Musées, Patrimoine et Culture scientifiques et techniques

$115 \mid 2008$

janvier - février 2008

\title{
En famille au musée : Essai de mise en pratique dans la définition d'un espace d'exposition permanente
}

Didier Julien-Laferrière

\section{(2) OpenEdition}

Journals

Édition électronique

URL : http://journals.openedition.org/ocim/276

DOI : 10.4000/ocim. 276

ISSN : 2108-646X

Éditeur

OCIM

Édition imprimée

Date de publication : 1 février 2008

Pagination : 10-11

ISSN : 0994-1908

Référence électronique

Didier Julien-Laferrière, « En famille au musée : Essai de mise en pratique dans la définition d'un espace d'exposition permanente », La Lettre de I'OCIM [En ligne], 115 | 2008, mis en ligne le 10 novembre 2010, consulté le 01 mai 2019. URL : http://journals.openedition.org/ocim/276 ; DOI : $10.4000 /$ ocim. 276

Ce document a été généré automatiquement le 1 mai 2019.

Tous droits réservés 


\title{
En famille au musée : Essai de mise en pratique dans la définition d'un espace d'exposition permanente
}

\author{
Didier Julien-Laferrière
}

1 Depuis 1994, année d'ouverture de la Grande Galerie de l'Évolution (GGE), les études réalisées sur le site par l'Observatoire permanent des Publics ${ }^{1}$ révèlent une augmentation régulière de la proportion du public familial : de moins de $30 \%$ du public individuel en 1995, celui-ci avoisine les $50 \%$ depuis 2000. Cette tendance suit un mouvement actuel général dans les musées et justifie que ceux-ci portent une attention particulière au public familial.

2 Parallèlement s'est révélé un certain manque dans l'offre de la GGE vis-à-vis des enfants d'âge moyen. Le spectacle des animaux naturalisés émerveille toujours les plus petits, alors que la richesse des contenus portés par les balcons périphériques répond à la curiosité des plus grands. Par contre, vis-à-vis des enfants d'âge correspondant grosso modo à l'école primaire, les informations sont rares dans la première partie de l'exposition puis complexes et difficiles d'accès dans les parties suivantes, et ce d'autant plus que ces enfants sont demandeurs d'autonomie de visite. L'espace conçu à leur intention, la "salle de découverte ", occupe une surface réduite et est situé en dehors du parcours principal de visite. De fait, les études de public révèlent qu'il passe inaperçu pour beaucoup de visiteurs et ceux qui visitent cet espace en ressortent peu satisfaits.

L'augmentation de cette catégorie de public et la nécessité pour l'institution de sty adapter a conduit le Muséum national d'Histoire naturelle à mettre en œuvre un nouvel espace d'exposition permanente pour les enfants au sein de la Grande Galerie de l'Évolution². Une question essentielle s'est alors rapidement posée: exposition pour l'enfant seul ou pour l'enfant accompagné - et en particulier de ses parents? Deux opinions diamétralement opposées peuvent être mises en avant. L'une plaide pour l'autonomie, souhaitant d'abord répondre à la demande exprimée par l'enfant et soutenir sa démarche personnelle ${ }^{3}$. L'autre met en avant d'une part les motivations à la visite exprimées par la cellule familiale dans son ensemble ${ }^{4}$, d'autre part l'intérêt de la 
« coéducation » ou encore de «l'éducation informelle» dans le contexte particulier du musée et de l'exposition'.

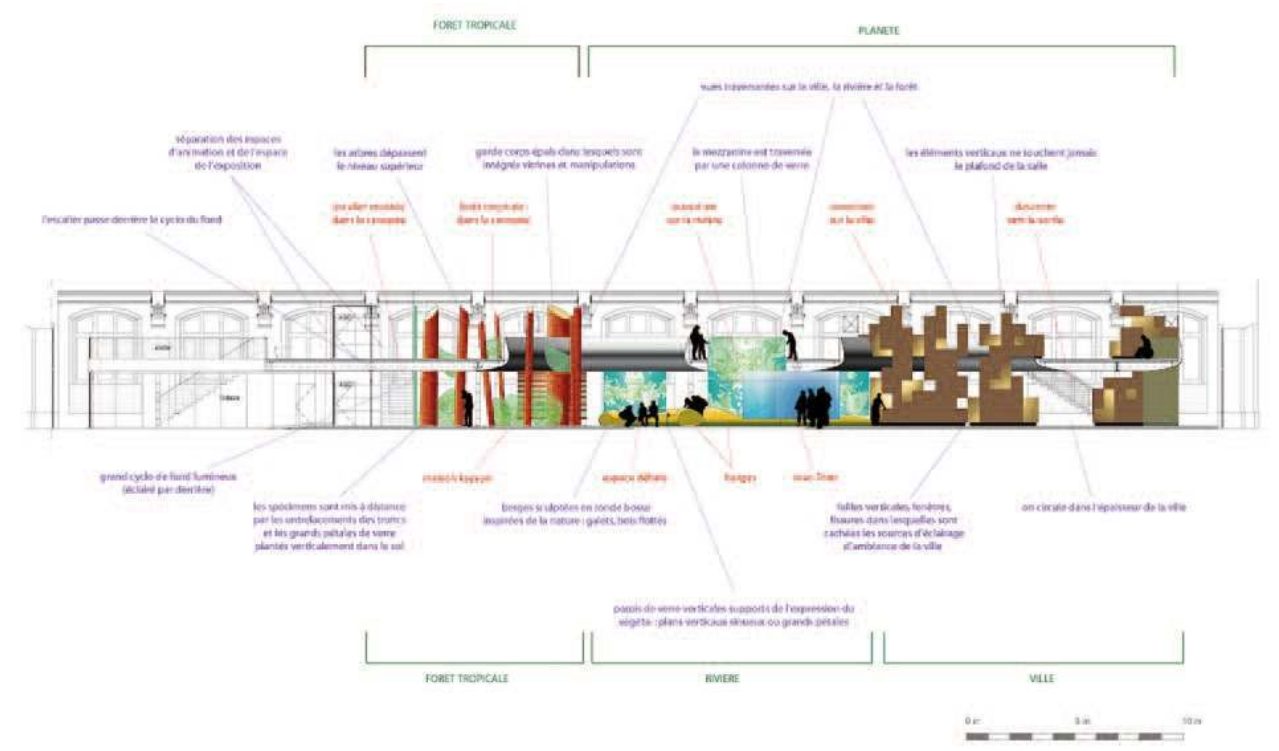

Coupe longitudinale de l'espace montrant dans la partie droite l'espace d'exposition et dans la partie gauche les deux salles d'activités. L'espace d'exposition est organisé en deux parties principales : au rez-de-chaussée, " Biodiversité », « Menaces » et « Actions » dans trois milieux, successivement la ville, la rivière et la forêt tropicale ; à l'étage, les mêmes thèmes sont abordés à l'échelle de la planète.

(c) P. Payeur / E. Lefrançois/M. Hardel / C. Le Bihan / A. Robaglia

En effet, l'une des principales motivation à la venue au musée en famille est le fait de se retrouver ensemble, de partager une activité commune, de se découvrir en dehors du cadre domestique. Par ailleurs, la coéducation au musée est reconnue comme contribuant à l'enrichissement de l'événement de visite comme à la socialisation de l'enfant ou à un partage égalitaire entre enfant et adulte de la connaissance et du plaisir d'apprendre.

C'est ce contexte de visite en famille qui a été privilégié dans l'élaboration du nouveau projet. De la même façon a été proposée la présence permanente d'une petite équipe d'animation chargée d'accompagner les groupes scolaires mais aussi, sous forme facultative, les visiteurs individuels. Cette équipe serait également chargée de définir et de mettre en œuvre de courtes animations, dites " animations-flash ", sur le lieu même de l'exposition, s'inspirant des pratiques de divers musées comme la Cité des Enfants ou l'Explor@dome à Paris'.

6 Les principes ayant conduit à la définition du projet et de ses formes de médiation se sont élaborés progressivement à partir de résultats de travaux de recherche et de réflexions théoriques mais aussi de choix pragmatiques inspirés d'expériences et de réalisations existantes ou issus de la sensibilité des différents membres de l'équipe projet, de l'identité de l'institution, ou encore des demandes de sa direction. Ils sont actuellement en cours de matérialisation sous forme d'un programme muséologique et d'un projet scénographique détaillés. L'ouverture de ce nouvel espace est prévue pour le printemps 2009. 


\section{NOTES}

1. Études réalisées annuellement de 1995 à 2001 par C. Fromont-Colin puis F. Lafon.

2. Proposition faite en 2003 par A. Parent qui assure depuis la conduite du projet.

3. Voir en particulier Singly, F. L'art et la manière de considérer un enfant dans un musée, colloque pARTages : quelles expositions d'art pour les enfants?, musée du Louvre, 28 avril 2006. Actes téléchargeables sur www.louvre.fr

4. Jonchéry, A. Quand la famille vient au musée: des pratiques de visites aux logiques culturelles. Thèse de doctorat en Muséologie, Muséum national d'Histoire naturelle, 2005

5. Voir par exemple Guichard, J. Nécessité d'une recherche éducative dans les expositions à caractère scientifique et technique, Publics \& Musées, n, 1995, pp. 95-115 ; Cordier, J.-P. et Serre, S., Interactions familiales au musée: approches sociologiques et psychocognitives, in La muséologie des sciences et ses publics, de J. Eidelman et M. Van-Praët (dir.), Paris : PUF, 2000, p. 259-279.

6. Selon des visites et interviews des membres du personnel de nombreux musées réalisées en 2003 et 2004 pour définir le projet.

INDEX

Mots-clés : enfants, exposition, famille, public

\section{AUTEUR}

DIDIER JULIEN-LAFERRIÈRE

Concepteur d'exposition au Muséum national d'Histoire naturelle 